\title{
De vuelta a la clínica. Métodos II. Arquitectura de la investigación clínica. Interacción sujeto, maniobras y enfermedad a través del tiempo
}

\author{
Juan O. Talavera, ${ }^{1 *}$ Ivonne A. Roy-García, ${ }^{2}$ Marcela Pérez-Rodríguez, ${ }^{2}$ Lino Palacios-Cruz ${ }^{3}$ y Rodolfo \\ Rivas-Ruíz² \\ ${ }^{1}$ Centro Médico ABC, Dirección de Enseñanza e Investigación; ${ }^{2}$ Instituto Mexicano del Seguro Social, Centro Médico Nacional Siglo XXI, Centro \\ de Adiestramiento en Investigación Clínica; ${ }^{3}$ Instituto Nacional de Psiquiatría "Dr. Ramón de la Fuente", Subdirección de Investigaciones Clínicas, \\ Departamento de Epidemiología Clínica. Ciudad de México, México
}

\section{Resumen}

La medicina se caracteriza por la aplicación del método científico a través del juicio clínico, por la correcta interpretación y el uso del curso clínico o historia natural de la enfermedad; su descripción más lograda la observamos en la arquitectura de la investigación clínica. A través de una secuencia temporal, este modelo explica el fenómeno de causalidad con tres apartados: estado basal, maniobra y desenlace. En el estado basal se evalúa quién es el paciente, de donde proviene, sus condiciones generales, el diagnóstico, el estadio y la agresividad de la patología, las complicaciones, terapias previas, nivel socioeconómico-cultural, hábitos, indicaciones o contraindicaciones terapéuticas y se prevé la evolución esperada. De la maniobra se pueden evaluar los factores de riesgo o pronóstico, tratamiento específico, sintomático y medidas generales. En el desenlace se vigila la evolución temprana y tardía. El modelo también permite determinar las causas de pérdida de seguimiento. Anticipar la evolución del paciente al reconocer su condición, enfermedad y efecto esperado de la decisiones médicas permite actuar anticipadamente, ya que esperar las manifestaciones del proceso evolutivo de la enfermedad resulta en detrimento del paciente.

PALABRAS CLAVE: Curso clínico. Juicio clínico. Investigación clínica. Causalidad.

\section{Back to the clinic. Methods II. Architecture of clinical research. Subject interaction, maneuvers and disease throughout time}

\section{Abstract}

Medicine is characterized by the application of the scientific method through clinical judgment, by correct interpretation and use of the clinical course and/or natural history of the disease; its best description is observed in the architecture of clinical research. Through a temporal sequence, this model explains the phenomenon of causality with three sections: baseline status, maneuver, and outcome. The baseline status assesses who the patient is, where does he come from, his general conditions, the diagnosis, stage and aggressiveness of the pathology, complications, previous therapies, socioeconomic-cultural level, habits, therapeutic indications or contraindications and the expected evolution is anticipated. In the maneuver, risk or prognostic factors, specific or symptom treatment, and general measures could be evaluated. In the outcome, early and late evolution are monitored. The model also allows the causes of follow-up loss to be determined. Anticipating patient evolution by recognizing his condition, disease, and expected effect of medical decisions allows acting in advance, since waiting for the manifestations of the evolutionary process of disease results in detriment to the patient.

KEY WORDS: Clinical course. Clinical judgment. Clinical research. Causality.

Correspondencia:

*Juan O. Talavera

E-mail: jotalaverap@abchospital.com
Gac Med Mex. 2020;156:438-446

Disponible en PubMed

www.gacetamedicademexico.com

0016-3813/@ 2020 Academia Nacional de Medicina de México, A.C. Publicado por Permanyer. Este es un artículo open access bajo la licencia CC BY-NC-ND (http://creativecommons.org/licenses/by-nc-nd/4.0/). 
Para entender el camino hay que andarlo, para hacerlo propio hay que recorrerlo una y otra vez.

Dicho POPULAR

\section{Introducción}

En la historia natural de la enfermedad podemosidentificar distintos componentes como el sujeto, el ambiente, las maniobras preventivas y la enfermedad. Al curso clínico habría que agregar las maniobras terapéuticas y las secuelas, muerte o curación, todos ellos coexistiendo en el tiempo. Este fenómeno clínico fue descrito por el doctor Alvan R. Feinstein bajo el concepto "arquitectura de la investigación clínica", que en su modelo de causalidad incluye tres componentes en una secuencia de tiempo: estado basal, maniobra y desenlace. Feinstein desarrolló su modelo a partir de una meticulosa descripción de la atención médica utilizando el método científico, lo que conocemos como "juicio clínico" (Figura 1) $\cdot^{1-3}$

En este artículo mostraremos la congruencia entre la práctica clínica y el modelo de arquitectura de la investigación clínica, y cómo ambos se corresponden con un artículo o protocolo de investigación.

\section{Causa de consulta (estado basal): demarcación diagnóstica y pronóstica}

Durante el quehacer clínico, el primer reto es la traducción de las manifestaciones referidas por el paciente en una agrupación conceptual inicial que permita tomar decisiones (diagnóstico sintomático, sindromático o etiológico). Este conjunto de manifestaciones subjetivas (síntomas) y objetivas (signos físi$\cos$, de laboratorio o gabinete) se obtiene durante la elaboración de la historia clínica, mediante el procedimiento denominado semiología clínica. ${ }^{4}$ Además de esta agrupación inicial, durante la elaboración de la historia clínica se recaba información que permite comprender a la persona con la que se trata. De esta forma, se estructura el estado basal del paciente al determinar quién es la persona (sus datos demográficos, antecedentes y hábitos) y la razón por la que asiste a consulta, ${ }^{5}$ es decir, su padecimiento.

De tal forma, en el estado basal (que se caracteriza por la evaluación inicial del paciente) se busca identificar quién es el paciente (sexo, edad, origen y comunidad donde habita y trabaja, su estrato socioeconómico y cultural, estilo de vida, hábitos y antecedentes hereditarios), sus condiciones generales (constitución y condición física, así como estado de ánimo), su diagnóstico (qué manifestaciones refiere y cómo se integran), el estadio, la agresividad de la patología, las complicaciones generadas por dicha patología, la comorbilidad que acompaña a la patología en estudio (con relación directa o no) o que ya presentaba previamente el paciente, así como los tratamientos previos a causa de la entidad clínica en estudio, por sus complicaciones o comorbilidad. Con esta historia clínica se decide el procedimiento diagnóstico-terapéutico, siempre considerando la expectativa de evolución de la enfermedad y las expectativas del paciente. Es decir, las decisiones se toman con el conocimiento del curso clínico de la enfermedad y las expectativas del paciente.

Al elaborar la historia clínica se puede identificar las características descritas en el modelo arquitectónico de la investigación, que en el estado basal incluyen lo que conocemos como demarcación diagnóstica y estratificación pronóstica.

En un proyecto de investigación, la demarcación diagnóstica (Figura 1, Tabla 1a) ${ }^{6}$ tiene inicialmente como objetivo promover la inclusión de una muestra que permita evaluar el efecto de la maniobra en la condición inicial y, de esta forma, evitar un ensamble inadecuado. Para lo anterior se requiere contar con cuatro requisitos:

1. Población con moderada a alta incidencia del desenlace de interés. Es importante recordar que las bajas incidencias requieren grandes muestras o tiempos muy prolongados de seguimiento, lo que dificulta formular una explicación causal de la maniobra.

2. Clara definición de la condición de base, con criterios específicos que establezcan el estado de salud o de enfermedad, según corresponda.

3. Que la población esté libre del desenlace en el momento de ser incluida y que exista la posibilidad de que este ocurra.

4. Que no exista indicación ni contraindicación previa para la maniobra por evaluar.

Por su parte, la demarcación pronóstica (Figura 1, Tabla $1 b)^{7}$ tiene como objetivo la identificación de la población con mayor probabilidad premaniobra de sufrir el desenlace y, en consecuencia, la decisión de las consideraciones terapéuticas específicas; además, desde el punto de vista metodológico se pueden realizar las estrategias pertinentes para evitar una percepción inadecuada del verdadero efecto de la maniobra principal y así evitar el sesgo de susceptibilidad. Estas estrategias son la estratificación de la población en su estado basal y el ajuste multivariado 


\section{EXPEDIENTE CLÍNICO}

Historia clínica Nota de indicaciones/estimación pronóstica

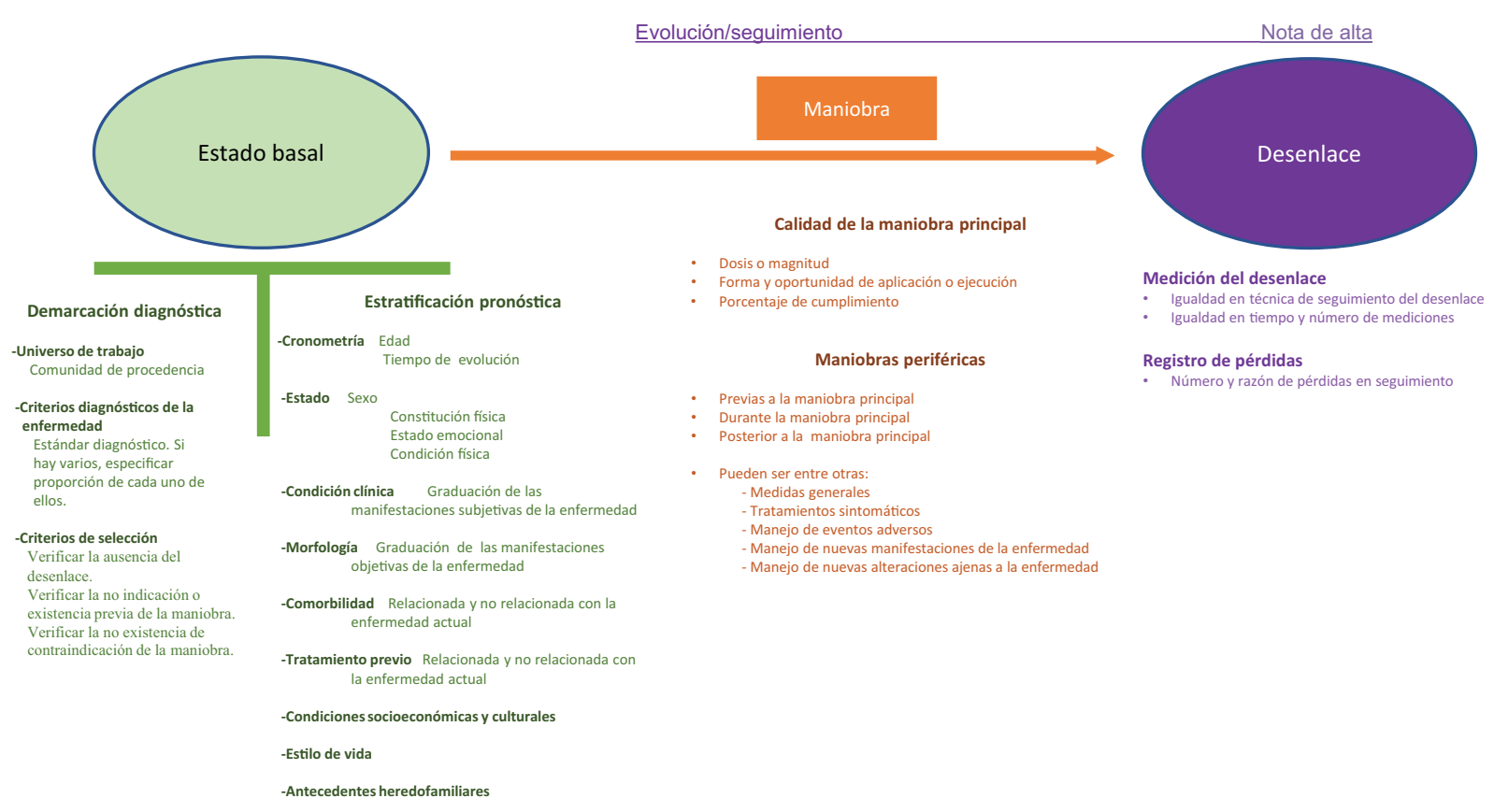

Figura 1. Curso clínico de la enfermedad (arquitectura de la investigación clínica). El estado basal evalúa quién es el paciente, de dónde proviene, condiciones generales, diagnóstico, estadio y agresividad de la patología, complicaciones, tratamientos previos, nivel socioeconómico y cultural, hábitos, indicaciones o contraindicaciones terapéuticas y evolución esperada. La maniobra evalúa factores de riesgo, factores pronóstico, tratamiento específico, sintomático y medidas generales. El desenlace incluye la evolución temprana y tardía y determina las causas de pérdida de seguimiento. Prever la evolución del paciente al reconocer su condición, enfermedad y efecto esperado de las decisiones terapéuticas permite actuar anticipadamente. Esperar las manifestaciones del proceso evolutivo de la enfermedad resulta en detrimento del paciente.

durante el análisis. Es necesario recordar que este sesgo no se evita con la aleatorización propia de los ensayos clínicos, ni aun cuando queden homogéneamente distribuidas todas las características entre las maniobras.

De igual forma, se debe reconocer que el efecto observado de la maniobra es el efecto promedio en la población predominante; por ejemplo, si en un estudio de pacientes con cáncer de mama tienen cabida pacientes con todos los estadios de la enfermedad, pero en estadios avanzados solo hay $5 \%$ de la población, el resultado se deberá a los pacientes en estadios iniciales, aun cuando en teoría en los criterios de selección se incluyó cualquier estadio de la enfermedad. Lo anterior se ha malinterpretado asumiendo que una maniobra es aplicable a quienes cubran los criterios de selección del estudio original, sin embargo, no es así, en realidad solo refleja el efecto en la población predominante. ${ }^{8}$ De igual forma, el ajuste durante el análisis solo muestra cuál es el resultado en una población promedio, es decir, cuando se controla o ajusta por una o más variables se realiza un procedimiento estadístico multivariado para mostrar el efecto de la maniobra principal, asumiendo que el resto de las características del paciente y de la enfermedad son constantes y que corresponden al promedio de dichas características en la población en estudio. En ambos casos, el resultado siempre estará mostrando lo que ocurre en la población predominante en la que se realizó el estudio.

\section{Decisiones terapéuticas o identificación de factores de riesgo o indicadores pronóstico: maniobra principal y periféricas}

Una vez caracterizado el paciente en el estado basal y con el conocimiento de la historia o curso clínico de la enfermedad debe tomarse una serie de decisiones. Inicialmente implica decisiones terapéuticas respecto a la patología en estudio; por un lado, puede ser un tratamiento específico dirigido a resolver el problema, es decir, un tratamiento con intención curativa; por otro lado, puede ser un tratamiento sintomático que busca 
Tabla 1a. Causa de consulta (estado basal): demarcación diagnóstica

\begin{tabular}{|c|c|c|c|c|}
\hline Práctica clínica & $\begin{array}{l}\text { Diseño } \\
\text { arquitectónico, } \\
\text { demarcación } \\
\text { diagnóstica }\end{array}$ & $\begin{array}{l}\text { Métodos de un } \\
\text { artículo/proyecto } \\
\text { de investigación }\end{array}$ & $\begin{array}{l}\text { Razonamiento común } \\
\text { para la investigación y la } \\
\text { clínica }\end{array}$ & $\begin{array}{l}\text { Ejemplo en un artículo de investigación } \\
\text { Day et al. }{ }^{6} \text { realizaron un ensayo clínico } \\
\text { para determinar si la combinación de } \\
\text { anfotericina + flucitosina o altas dosis de } \\
\text { fluconazol o anfotericina mejoraban la } \\
\text { supervivencia de los pacientes con meningitis } \\
\text { por criptococo** }\end{array}$ \\
\hline $\begin{array}{l}\text { Comunidad donde } \\
\text { habita el paciente }\end{array}$ & $\begin{array}{l}\text { Selección de la } \\
\text { población }\end{array}$ & $\begin{array}{l}\text { Universo de } \\
\text { trabajo }\end{array}$ & $\begin{array}{l}\text { Identifica a una } \\
\text { población susceptible } \\
\text { del desenlace de } \\
\text { interés; localización } \\
\text { geográfica donde es } \\
\text { común el fenómeno, } \\
\text { o de moderada a alta } \\
\text { prevalencia de factores } \\
\text { de riesgo o pronóstico }\end{array}$ & $\begin{array}{l}\text { El universo de trabajo fueron pacientes con } \\
\text { infección por virus de la inmunodeficiencia } \\
\text { humana con diagnóstico de meningitis por } \\
\text { criptococo, atendidos en el Hospital de } \\
\text { Enfermedades Tropicales en Vietnam. La } \\
\text { población seleccionada es susceptible de } \\
\text { presentar el desenlace, con una mortalidad } \\
\text { anual mayor a } 60 \%\end{array}$ \\
\hline $\begin{array}{l}\text { Porque nos consulta } \\
\text { el paciente. Qué } \\
\text { tiene y cómo se hizo } \\
\text { el diagnóstico (qué } \\
\text { enfermedad padece) }\end{array}$ & $\begin{array}{l}\text { Definición del } \\
\text { diagnóstico }\end{array}$ & $\begin{array}{l}\text { Criterios } \\
\text { diagnósticos } \\
\text { específicos de } \\
\text { la condición en } \\
\text { estudio }\end{array}$ & $\begin{array}{l}\text { Identifica el diagnóstico } \\
\text { y los criterios con } \\
\text { los cuales se realizó. } \\
\text { (ejemplo, diabetes } \\
\text { mellitus tipo } 2 \\
\text { determinada por } \\
\text { hemoglobina glucosilada, } \\
\text { glucosa en ayunas o } \\
\text { prueba de tolerancia a la } \\
\text { glucosa) }\end{array}$ & $\begin{array}{l}\text { El diagnóstico de meningitis por criptococo } \\
\text { fue realizado en quienes presentaron signos } \\
\text { y síntomas de meningitis por criptococo y, } \\
\text { además, cumplían con alguna de las siguientes } \\
\text { condiciones: } \\
\text { 1. Tinción con tinta china positiva en líquido } \\
\text { cefalorraquídeo. } \\
\text { 2. Antígeno para criptococo positivo en líquido } \\
\text { cefalorraquídeo. } \\
\text { 3. Cultivo de líquido cefalorraquídeo positivo } \\
\text { para Cryptococcus neoformans. } \\
\text { 4. Hemocultivo positivo para Cryptococcus } \\
\text { neoformans (título > 1:10). }\end{array}$ \\
\hline $\begin{array}{l}\text { Condición del } \\
\text { paciente y } \\
\text { características } \\
\text { de la enfermedad } \\
\text { que indican o } \\
\text { contraindican } \\
\text { un tratamiento } \\
\text { específico }\end{array}$ & $\begin{array}{l}\text { Criterios de } \\
\text { selección* }\end{array}$ & $\begin{array}{l}\text { Criterios de } \\
\text { selección. } \\
\text { Diagrama de flujo } \\
\text { de los pacientes } \\
\text { finalmente } \\
\text { seleccionados }\end{array}$ & $\begin{array}{l}\text { Garantiza la presencia } \\
\text { de sujetos con } \\
\text { posibilidad de presentar } \\
\text { el desenlace y protege } \\
\text { de no incluir a quienes } \\
\text { ya tienen indicación } \\
\text { o contraindicación de } \\
\text { alguna de las maniobras }\end{array}$ & $\begin{array}{l}\text { Como criterios de no inclusión absolutos se } \\
\text { consideraron creatinina elevada, falla renal } \\
\text { o hepática, embarazo y/o administración de } \\
\text { rifampicina, que contraindican la asignación de } \\
\text { la maniobra. No se incluyó a quienes ya tenían } \\
\text { indicación de la maniobra con anterioridad, como } \\
\text { el antecedente de tratamiento antifúngico por más } \\
\text { de tres días, que podría influir en el desenlace }\end{array}$ \\
\hline
\end{tabular}

*Los criterios de selección dan continuidad al diagnóstico de la condición basal y definen quién es candidato para participar en un proyecto específico. Pueden ser absolutos o relativos. Los criterios absolutos son ineludibles y están relacionados con la exposición a la maniobra o a la posible ocurrencia del desenlace. Los relacionados con el desenlace se refieren a quienes no deben entrar al estudio porque ya existe el desenlace o por imposibilidad para presentarlo, y los relacionados con la maniobra se refieren a quienes no deben entrar porque existe la indicación o contraindicación previa de esta. Los criterios relativos son evitables y en general se consideran para reducir el tamaño de muestra, tiempo y recursos. Sin embargo, en ocasiones no se limita la entrada y estos criterios relativos deben ser considerados para estratificar a la población por el mayor o menor riesgo de sufrir el desenlace (demarcación pronóstica) o para realizar el ajuste en el análisis.

${ }^{*}$ Si bien no se consideró la gravedad del cuadro clínico (criterio relativo) como criterio de inclusión, debe ser tomada en cuenta al analizar los resultados y debe ser incluida en los parámetros de la estratificación pronóstica.

mitigar las molestias del paciente; $y$, finalmente, medidas generales que dan soporte al paciente para enfrentar la enfermedad y las terapias junto con sus eventos adversos. Desde luego se agregan decisiones terapéuticas para el manejo específico de eventos adversos, complicaciones propias de la enfermedad o entidades clínicas independientes a esta, pero que presenta el paciente durante el curso clínico de la enfermedad. En todos estos caso se debe considerar y calcular el pronóstico del paciente $y$, aun cuando esté libre de la enfermedad, cuál o cuáles son los factores de riesgo para desarrollarla.
Identificar las decisiones terapéuticas como acciones que modifican el estado basal del paciente es habitual, sin embargo, existen acciones realizadas por el paciente o por la naturaleza que de igual manera lo modifican, las cuales identificamos como factores de riesgo cuando ocurren antes de la enfermedad o como indicadores pronósticos cuando siguen a la enfermedad (por ejemplo, por acción de la naturaleza [edad], acciones propias del paciente [tabaquismo] 0 acciones por otras indicaciones terapéuticas ajenas a la patología actual del paciente, pero que de igual forma pueden impactar en el desenlace). 
Tabla 1b. Causa de consulta (estado basal): demarcación pronóstica

\begin{tabular}{|c|c|c|c|c|}
\hline Práctica clínica & $\begin{array}{l}\text { Diseño } \\
\text { arquitectónico } \\
\text { Demarcación } \\
\text { pronóstica }\end{array}$ & $\begin{array}{l}\text { Proyecto de } \\
\text { investigación/ } \\
\text { artículo }\end{array}$ & Razón & $\begin{array}{l}\text { Ejemplo en un artículo de investigación } \\
\text { Sun et al. realizaron un estudio cuyo } \\
\text { objetivo fue evaluar la asociación } \\
\text { entre el nivel de ingesta de } \\
\text { vitamina D y el desarrollo de enfermedad } \\
\text { cardiovascular* }\end{array}$ \\
\hline $\begin{array}{l}\text { - Quién es el paciente } \\
\text { (sexo, edad, estrato } \\
\text { socioeconómico y cultural, } \\
\text { estilo de vida y hábitos). } \\
\text { - Condiciones generales del } \\
\text { paciente (constitución y } \\
\text { condición física, estado de } \\
\text { ánimo). } \\
\text { - Estadio y agresividad de la } \\
\text { patología. } \\
\text { - Complicaciones generadas } \\
\text { por la patología. } \\
\text { - Comorbilidad que } \\
\text { acompaña a la patología o } \\
\text { previamente presente en el } \\
\text { paciente. } \\
\text { - Tratamientos previos a } \\
\text { causa de la patología en } \\
\text { estudio o por comorbilidad } \\
\text { previa. }\end{array}$ & $\begin{array}{l}\text { Estratificación: } \\
\text { - Demografía. } \\
\text { - Cronometría. } \\
\text { - Estado. } \\
\text { - Condición clínica. } \\
\text { - Morfología. } \\
\text { - Complicaciones. } \\
\text { - Comorbilidades. } \\
\text { - Tratamientos } \\
\text { previos. } \\
\text { - Nivel } \\
\text { socioeconómico y } \\
\text { cultural. } \\
\text { - Estilo de vida y } \\
\text { hábitos. } \\
\text { - Antecedentes } \\
\text { heredofamiliares }\end{array}$ & $\begin{array}{l}\text { Descripción } \\
\text { operativa y } \\
\text { conceptual de } \\
\text { las variables } \\
\text { de confusión o } \\
\text { modificadoras, } \\
\text { presentes en el } \\
\text { estado basal }\end{array}$ & $\begin{array}{l}\text { Controlar el efecto } \\
\text { de variables ajenas } \\
\text { a la principal o ya } \\
\text { presentes desde } \\
\text { el estado basal, } \\
\text { de tal manera que } \\
\text { se pueda aislar el } \\
\text { efecto real de la } \\
\text { misma }\end{array}$ & $\begin{array}{l}\text { Al evaluar las características del estado } \\
\text { basal se identifican los siguientes } \\
\text { elementos de la estratificación pronóstica: } \\
\text { - Datos demográficos: sexo, lugar de } \\
\text { residencia con exposición solar. } \\
\text { - Cronometría: edad. } \\
\text { - Estado: estado nutricional, índice de } \\
\text { masa corporal. } \\
\text { - Estratificación clínica y morfológica: eran } \\
\text { sujetos sanos. } \\
\text { - Tratamiento previo: uso de aspirina o } \\
\text { tratamiento multivitamínico. } \\
\text { - Comorbilidad: antecedente de diabetes, } \\
\text { hipertensión y dislipidemia. } \\
\text { - Estilo de vida y hábitos: tabaquismo, } \\
\text { alcoholismo, ingesta calórica total y } \\
\text { actividad física. } \\
\text { - Estratificación por herencia: antecedente } \\
\text { familiar de enfermedad cardiovascular. }\end{array}$ \\
\hline
\end{tabular}

Todas estas decisiones $u$ acciones se encuentran referidas en el modelo arquitectónico de la investigación, en el apartado de maniobras, las cuales pueden estratificarse como maniobra principal y maniobras periféricas (Figura 1, Tabla 2). La maniobra principal se refiere a la acción que representa el principal interés en la pregunta de investigación, de la cual debe hacerse una descripción de su correcta ejecución que permita medir la calidad, incluyendo el grado de adherencia. Aunado a esto se deben identificar todas las maniobras periféricas, es decir, las acciones o cambios de condición que se presentan entre el estado basal y el desenlace que pueden afectar a este último. El cuidado de estas dos características permite evitar o medir la magnitud del sesgo de ejecución, y señalar las diferencias generadas por distintos niveles de calidad entre las maniobras que se van a comparar o por la utilización desigual de maniobras adicionales entre los grupos. ${ }^{3} \mathrm{Al}$ respecto, es preocupante que numerosos ensayos clínicos atribuyan el desenlace solo a la maniobra principal, incluso en casos con una distancia de 10 años entre la maniobra y el desenlace, ignorando el conjunto de maniobras periféricas. ${ }^{9,10}$

\section{Evolución del paciente: el desenlace}

Todo paciente sometido a maniobras terapéuticas debe contar con evaluaciones repetidas durante el seguimiento, que permitan medir la respuesta inicial (un regulador intermedio por lo general), la respuesta final (desenlace) y la seguridad del paciente (previsión y medición de eventos adversos). Durante el seguimiento, cada vez que un paciente deja de asistir a sus evaluaciones preocupa cuál es su estado de salud, si ya se resolvió el problema o si habrá empeorado y por eso dejo de asistir, o si simplemente lo olvidó o decidió buscar atención médica en otro lugar; en estos casos una llamada telefónica puede ayudar a identificar la causa. Todas estas mediciones están consideradas en el desenlace del modelo arquitectónico, bajo el concepto de medición ideal estandarizada del desenlace y eventos adversos y registro de pérdidas durante el seguimiento.

La medición ideal estandarizada del desenlace implica que se realice la evaluación de todos los pacientes bajo los mismos criterios, de preferencia los aceptados en forma internacional, y al mismo tiempo que el seguimiento, con el objetivo de evitar que una 
Tabla 2. Decisiones terapéuticas o identificación de factores de riesgo o indicadores pronóstico: maniobras principal y periféricas

\begin{tabular}{|l|l|l|} 
Práctica clínica Diseño arquitectónico & $\begin{array}{l}\text { Proyecto de Razón } \\
\text { investigación/ } \\
\text { artículo }\end{array}$ \\
\hline
\end{tabular}

Tratamiento específico. Factor de riesgo/ pronóstico
La maniobra puede ser un tratamiento, pero también un factor de riesgo o factor pronóstico.* Se evalúa la calidad de la maniobra principal (intensidad, frecuencia, continuidad [adherencia], forma de aplicación)

Tratamiento sintomático Maniobras periféricas. y de medidas generales, Todo suceso que así como todas aquellas otras maniobras para tratar eventos adversos, y complicaciones que se presenten durante el curso de la enfermedad, estén o no relacionadas con esta, o bien, todo suceso que ocurra durante el periodo de seguimiento y que pueda impactar en la evolución del paciente ocurre alrededor 0 acompañando a la maniobra principal y que puede influir en el desenlace
Maniobra de interés, también conocida como variable principal o variable independiente

Variables de confusión (relacionadas con el desenlace y la maniobra principal) o modificadoras (relacionadas solo con el desenlace). Estas siguen siendo variables independientes
Ofrece los parámetros que miden la exposición a la maniobra principal; calidad de la maniobra
Ejemplo en un artículo de investigación Aggarwal et al. ${ }^{10}$ publicaron un ensayo clínico aleatorizado ciego sencillo. cuyo objetivo fue evaluar la eficacia, seguridad y tolerabilidad de $2000 \mathrm{mg}$ diarios de metformina XR una vez al día versus metformina IR dos veces al día en pacientes con diabetes mellitus tipo 2 vírgenes a tratamiento farmacológico**

Los participantes fueron aleatorizados a una de las dos maneras de recibir $2000 \mathrm{mg}$ de metformina:

1) 2000 mg de metformina $X R$ una vez al día junto con los alimentos de la tarde.

2) $1000 \mathrm{mg}$ de metformina IR dos veces al día, en la mañana y en la tarde junto con los alimentos. Ambos tratamientos por 24 semanas

Acompañando a la Los participantes, adicionalmente al maniobra principal tratamiento farmacológico, recibían asesoría existe una serie de acciones que sobre estilo de vida y alimentación de igual forma pueden influir en el desenlace y confundir respecto al efecto de la maniobra principal. En consecuencia, deben ser consideradas para aislar el efecto real de la maniobra principal

*Maniobra, acción que se espera modifique la condición basal: a) Experimental, maniobra asignada con fines de investigación. b) Observacional, maniobra presente por causas ajenas a la investigación (por ejemplo, por acción de la naturaleza [edad], autoseleccionada [tabaquismo], por otras indicaciones [atención médica]). Identificar la calidad de la maniobra permite realizar el análisis por intención a tratamiento (IT) y el análisis por protocolo (PP). A mayor apego, mayor la probabilidad de ver el efecto real de la maniobra. Recordemos que el análisis IT se refiere al análisis de todos los pacientes en el grupo asignado, hayan recibido o no la maniobra en forma adecuada. El análisis PP se refiere al análisis solo de pacientes cuya maniobra fue ejecutada en forma adecuada.

${ }^{*}$ Aun cuando ambos grupos están recibiendo la misma dosis del fármaco y se ajustan por múltiples interacciones y valores basales de hemoglobina glucosilada, no se toma en consideración durante el análisis multivariado la dieta y otras variables del estilo de vida que han mostrado tener un efecto relevante en el control glucémico de los pacientes con diabetes mellitus tipo 2.

evaluación diferencial afecte el resultado y genere lo que conocemos como sesgo de detección (Figura 1, Tabla 3a). ${ }^{11,12}$ Siempre es indispensable la identificación de los eventos adversos; prácticamente no hay acción que solo tenga beneficios, de tal manera que siempre habrá que considerar el impacto adverso de la acción e, incluso, restar el valor del beneficio cuando el evento adverso sea equivalente o de mayor relevancia clínica. ${ }^{13}$

De igual forma, en la medición del desenlace debe considerarse el número y la razón de los pacientes perdidos durante el seguimiento. Las pérdidas no debidas al azar, sino por razones propias al tratamiento y a la enfermedad se asocian a la presencia del sesgo de transferencia, común en los estudios cuya pesquisa de la población es discordante con el estado basal estudios de casos, controles y encuesta transversal y series de casos, que frecuentemente se confunden con supuestas cohortes-, debido a que cuando se inicia el estudio solo se cuenta con sobrevivientes y no hay forma de medir la magnitud del error; mientras mayor sea el número de pacientes perdidos durante el seguimiento, mayor será la posibilidad y magnitud del error. En los estudios en los que la pesquisa coincide con el ensamble, como el ensayo clínico y la cohorte, es posible medir la magnitud del error dado que se conocen 
Tabla 3a. Evolución del paciente: desenlace primario, secundario y eventos adversos

\begin{tabular}{|l|l|l|}
\hline Práctica clínica Diseño arquitectónico & $\begin{array}{l}\text { Proyecto de } \\
\text { investigación/ } \\
\text { artículo }\end{array}$ & Razón \\
\hline
\end{tabular}

Seguimiento estandarizado al paciente de acuerdo con el curso clínico de la enfermedad (evaluación de desenlaces intermedios y la aparición o no del desenlace primario y en ocasiones los evaluaciones permiten modificar la terapia de ser necesario secundarios). Estas
Se trata de la medición del desenlace primario con evaluaciones iguales y con el estándar diagnóstico, preferentemente evaluaciones a ciegas
Se trata de la medición del desenlace primario, o variable dependiente. También se puede considerar la medición de otros desenlaces secundarios, pero estos siempre serán exploratorios

Eventos adversos

\section{Se vigilan datos de} alarma que pueden representar un riesgo a la vida del paciente 0 la necesidad de ajuste al tratamiento
Se requiere definir el desenlace de la forma más precisa posible y a tiempos específicos. La medición inapropiada y fuera de tiempo puede sub o sobre diagnosticar y generar diferencias artificiales entre los grupos. A mayor número de visitas en alguno de los grupos, mayor probabilidad de detectar el desenlace generando sesgos (el mayor número de visitas puede ser generado por mayores eventos adversos, ajuste de dosis o sospecha diagnóstica, entre otros)

La vigilancia y medición de los eventos adversos o complicaciones es un dato de alarma que permite mejorar el cuidado del paciente y que obliga a restar beneficio a la maniobra principal cuando dichos eventos ponen en riesgo la vida del paciente, o cuando el daño es superior al beneficio esperado

\section{Ejemplo en un artículo de investigación}

March et al. ${ }^{11}$ publicaron en 2004

un ensayo clínico denominado

Treatment for Adolescents with Depression Study (TADS), el cual tuvo como objetivo evaluar la efectividad de cuatro modalidades de tratamiento para niños y adolescentes con trastorno depresivo mayor*

Los desenlaces primario y secundario evaluaron la mejoría de los síntomas de depresión e ideación suicida a través de las siguientes escalas:

- CDRS, Children's Depression

Rating Scale Revised.

- CGI, Clinical Global Impressions.

- RADS, Reynolds Adolescent

Depression Scale, instrumento de autorreporte.

- SIQ-Jr, Suicidal Ideation

Questionnaire-Junior High School Version.

Las evaluaciones del desenlace se llevaron a cabo a las seis y 12 semanas en cada uno de los grupos de tratamiento

Entre los eventos adversos evaluados se encontró presencia de manía, hipomanía, irritabilidad, agitación, ansiedad, fatiga, sedación, conducta suicida y autolesiones

*La conducta suicida y las autolesiones si bien existían en un porcentaje de pacientes desde el inicio del estudio y se midió su reducción como un desenlace secundario, también hay que considerar la posibilidad de que aparezcan, por lo que resulta difícil distinguir si son parte de la historia natural de la enfermedad o del curso clínico al iniciar el tratamiento antidepresivo; en ocasiones pueden considerarse como un evento adverso, de ser así se observa una clara reducción con el tratamiento. Entre otros aspectos, la medición del desenlace fue a las seis y 12 semanas, lo cual es congruente con el tiempo necesario para observar respuesta en niños y adolescentes, a diferencia de los adultos en quienes ocurre en forma más temprana.

las pérdidas y el momento en que se presentaron (Figura 1, Tabla 3b)..$^{14,15}$

\section{Tiempos en la historia natural o curso clínico de la enfermedad: cronograma de actividades}

El conocimiento de la historia natural y del curso clínico de la enfermedad ofrece una estimación de la evolución esperada del paciente (Figura 2). A partir de este conocimiento se toman las distintas decisiones en forma anticipada, tanto diagnósticas como terapéuticas, previendo la posible evolución del paciente; no se espera a que un suceso progrese, momento en el cual las acciones pueden tener menor beneficio. El conocimiento del curso clínico en un proyecto de investigación 0 artículo publicado se refleja en el cronograma de actividades, el cual permite planear y registrar la 
Tabla 3b. Evolución del paciente: pérdida de seguimiento

$\begin{array}{llll}\text { Práctica clínica } & \begin{array}{l}\text { Diseño } \\ \text { arquitectónico }\end{array} & \begin{array}{l}\text { Proyecto de } \\ \text { investigación/artículo }\end{array} & \text { Razón }\end{array}$

Cada vez que se pierde el seguimiento de un paciente, debe considerarse la posibilidad de que se trate de falla terapéutica, complicación o evento adverso. Aunque también puede ser por resolución del problema o búsqueda de atención en otro lugar

\section{seguimiento. \\ Identificar la caus \\ permite estimar \\ el resultado a favo \\ pacientes}

$\begin{array}{lll}\text { Pérdida de } & \text { Diagrama de flujo } & \text { Documentar } \\ \text { seguimiento. } & \text { de los pacientes } & \text { las pérdidas es } \\ \text { Identificar la causa } & \text { perdidos durante el } & \text { indispensable para } \\ \text { permite estimar } & \text { seguimiento. Suele } & \text { identificar el verdadero } \\ \text { la posibilidad de } & \text { estar integrado al } & \text { éxito de las acciones } \\ \text { tener un desvío en } & \text { diagrama inicial } & \text { médicas, y para } \\ \text { el resultado a favor } & \text { que acompaña a } & \text { evitar sobreestimar o } \\ \text { de alguno de los } & \text { la selección de los } & \text { subestimar el resultado } \\ \text { grupos } & \text { pacientes } & \text { de estas }\end{array}$

\section{Ejemplo en un artículo de investigación}

Miguel et al.., en 2018, ${ }^{14}$ publicaron los resultados de un estudio que tuvo el objetivo de evaluar la efectividad de un algoritmo de tratamiento para trastorno bipolar (TBP), usando las alternativas terapéuticas disponibles en el sistema de salud brasileño*

El estudio incluyó de forma inicial a 107 pacientes. A la semana 8 de seguimiento asistieron solo 88 participantes y a la semana 16 solo 84 participantes, lo que representó una pérdida de seguimiento de $21 \%$ del total de participantes.

Entre las causas de pérdida del seguimiento se encontraron el abandono y la presencia de eventos adversos al tratamiento

*Un suceso común es encontrar en los artículos solo los datos de los pacientes que permanecieron hasta el final del estudio; de hecho, en muchos artículos no se menciona cuál era el tamaño inicial de la población. En este trabajo, la muestra original fue de 107 pacientes, de lo cuales solo 84 permanecieron hasta las 16 semanas. En cada tabla del análisis del curso clínico/historia natural de la enfermedad debe mostrarse el total de pacientes existentes hasta ese momento. Deben realizarse comparaciones que muestren si los pacientes perdidos en sus últimas mediciones se parecen a los que continúan, lo que permitiría estimar la magnitud del sesgo de transferencia.

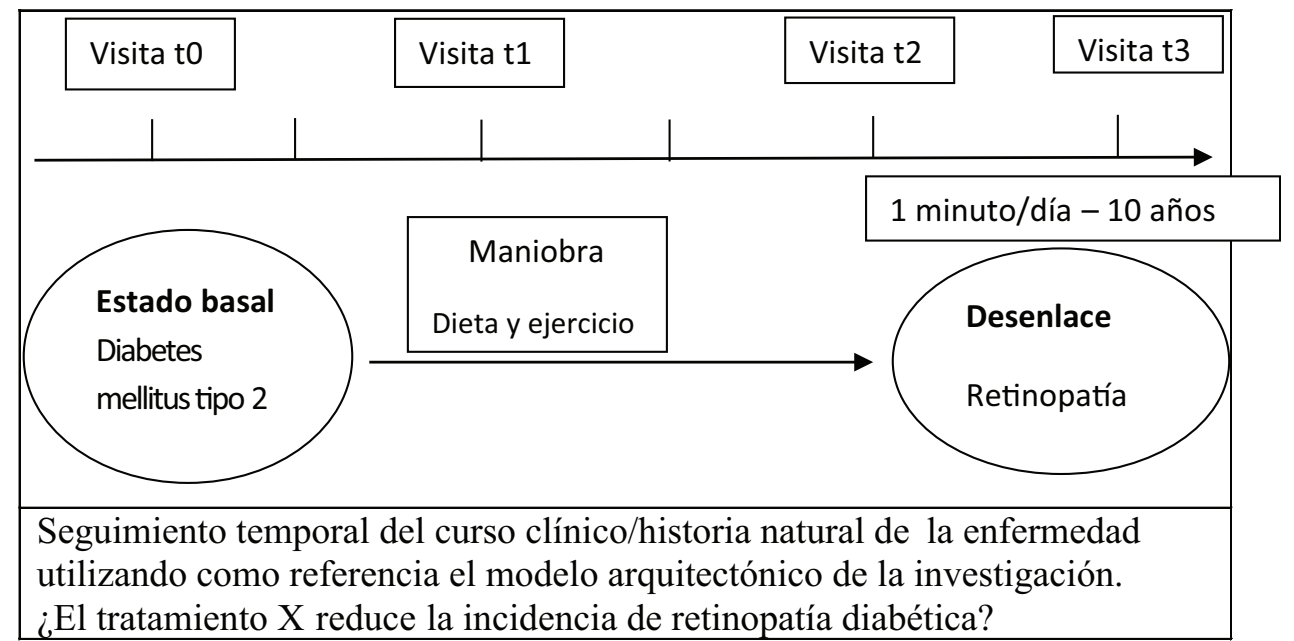

\begin{tabular}{|c|c|c|c|c|c|c|c|c|}
\hline $\begin{array}{l}\text { Característica por } \\
\text { registrar }\end{array}$ & $\begin{array}{c}\text { to } \\
\text { Visita } 1 \\
\text { Mes } 0\end{array}$ & $\begin{array}{c}\text { to } \\
\text { Visita } 2 \\
\text { Mes } 1\end{array}$ & $\begin{array}{c}\text { t1 } \\
\text { Visita } 3 \\
\text { Mes } 3\end{array}$ & $\begin{array}{c}\text { t1 } \\
\text { Visita } 4 \\
\text { Mes } 6\end{array}$ & $\begin{array}{l}\text { t1, t2, t3 } \\
\text { Visita } 5 \\
\text { Mes } 12\end{array}$ & $\begin{array}{c}\text { t1 } \\
\text { Visita } 6 \\
\text { Mes } 18\end{array}$ & $\begin{array}{l}\text { t1, t2, t3 } \\
\text { Visita } 7 \\
\text { Mes } 24\end{array}$ & $\begin{array}{l}\text { t1, t2, t3 } \\
\text { Visita } 8 \\
\text { Mes } 36\end{array}$ \\
\hline Edad & $x$ & - & - & - & - & - & - & - \\
\hline Sexo & $x$ & - & - & - & - & - & - & - \\
\hline Tiempo de evolución & $x$ & - & - & - & - & - & - & - \\
\hline Índice de masa corporal & $x$ & $x$ & $x$ & $x$ & $x$ & $x$ & $x$ & $x$ \\
\hline Glucosa & $x$ & - & - & $x$ & $x$ & $x$ & $x$ & $x$ \\
\hline Dieta y ejercicio & $x$ & $x$ & $x$ & $x$ & $x$ & $x$ & $x$ & $x$ \\
\hline Alteraciones de la visión & - & - & - & - & $x$ & - & $x$ & $x$ \\
\hline Retinopatía diabética & $x$ & - & - & - & $x$ & - & $x$ & $x$ \\
\hline
\end{tabular}

Figura 2. Cronograma de actividades (conforme la arquitectura de la investigación clínica). Las visitas to, 11 , t2 y t3 están relacionadas con el modelo arquitectónico; to = estado basal, $t 1$ = maniobra, $t 2=$ respuesta temprana a la maniobra y posibles eventualidades, $t 3=$ desenlace. Las mediciones en los tiempos 1, 2 y 3 se pueden sobreponer durante el seguimiento y realizarse múltiples veces 1.1, 2.1, 3.1, 1.2, 2.2, 3.2... 
secuencia temporal con la que se presentan los diferentes componentes del fenómeno de causalidad.

\section{Comentario final}

Podemos terminar destacando que la información contenida en los artículos de investigación refleja el quehacer clínico; su lectura nos ofrece un recorrido por la historia natural o el curso clínico de una enfermedad. La aplicación del modelo arquitectónico de la investigación en la lectura crítica de estos textos permite examinar el fenómeno de causalidad en sus diferentes componentes, identificar la temporalidad en la que aparecen, la contribución de cada uno y reconocer cuáles son factibles de ser modificados. La reflexión consciente de este conocimiento cada vez que se enfrentan las dolencias de los pacientes permite tomar decisiones objetivas; prever la evolución del paciente a partir de reconocer su condición, su enfermedad y las consecuencias de las decisiones diagnóstico-terapéuticas permite actuar anticipadamente y reducir el daño en el paciente. Esperar a responder hasta que los distintos sucesos ocurran resultará en detrimento de la salud del paciente.

Cada vez que leemos un artículo o diseñamos y ejecutamos un proyecto de investigación estamos recreando el fenómeno de causalidad con el que convivimos día a día en la práctica clínica. Integrar a nuestra atención médica el entendimiento y quehacer de la investigación incrementa nuestra experiencia para comprender este fenómeno y acorta el tiempo para ser un experto en un área cualquiera. No debemos desperdiciar el aprendizaje a partir de la experiencia de otros; depender solo de nuestra experiencia nos llevará muchos años antes de actuar de la mejor forma posible. Si bien nunca dominaremos por completo el tema clínico de nuestro interés (por ejemplo, diabetes mellitus tipo 2, epilepsia, esclerosis múltiple, etcétera), acortaremos el camino al dominio del tema al adjuntar a nuestro quehacer clínico la lectura estructurada, el entendimiento y la participación en el proceso de investigación.

Finalmente, debemos retomar la expresión del doctor Alvan R. Feinstein, quien refiere que en el modelo arquitectónico no hay nada que un médico sensato no sepa 0 no pueda entender. ${ }^{16}$ Para entender el camino hay que andarlo, para hacerlo propio hay que recorrerlo una y otra vez.

\section{Conflicto de intereses}

Los autores declaran no tener conflicto de interés alguno.

\section{Financiamiento}

Los autores no recibieron patrocinio para llevar a cabo este artículo.

\section{Responsabilidades éticas}

Protección de personas y animales. Los autores declaran que para esta investigación no se realizaron experimentos en seres humanos ni en animales.

Confidencialidad de los datos. Los autores declaran que en este artículo no aparecen datos de pacientes.

Derecho a la privacidad y consentimiento informado. Los autores declaran que en este artículo no aparecen datos de pacientes.

\section{Bibliografía}

1. Feinstein AR. Clinical epidemiology: the architecture of clinical research. EE. UU.: Saunders; 1985

2. De la Sierra T. El método científico aplicado a la clínica. México: Méndez Editores; 2011.

3. Talavera JO, Wacher-Rodarte NH, Rivas-Ruiz R. Investigación clínica III. Estudios de causalidad. Rev Med Inst Mex Seguro Soc. 2011;49:289-294.

4. Casademont J. Fisiopatología y semiología clínica. ¿Dónde estamos y hacia dónde deberíamos dirigirnos? Educ Med. 2018:19:48-50.

5. Feinstein AR. Boolean algebra and clinical taxonomy. I. Analytic synthesis of the general spectrum of a human disease. $\mathrm{N}$ Engl $\mathrm{J}$ Med. 1963;269:929-938.

6. Day JN, Chau TT, Wolbers M, Mai PP, Dung NT, Mai NH, et al. Combination antifungal therapy for cryptococcal meningitis. $\mathrm{N}$ Engl $\mathrm{J}$ Med. 2013;368:1291-1302.

7. Sun $Q$, Shi L, Rimm EB, Giovannucci EL, Hu FB, Manson JE, et al. Vitamin $D$ intake and risk of cardiovascular disease in US men and women. Am J Clin Nutr. 2011;94:534-542.

8. Hurvitz SA, Martin M, Symmans WF, Jung KH, Huang CS, Thompson AM, et al. Neoadjuvant trastuzumab, pertuzumab, and chemotherapy versus trastuzumab emtansine plus pertuzumab in patients with HER2-positive breast cancer (KRISTINE): a randomised, open-label, multicentre, phase 3 trial. Lancet Oncol. 2018;19:115-126.

9. Holman RR, Paul SK, Bethel MA, Matthews DR, Neil HA. 10-year follow-up of intensive glucose control in type 2 diabetes. $\mathrm{N}$ Engl $\mathrm{J}$ Med. 2008:359:1577-1589.

10. Aggarwal N, Singla A, Mathieu C, Montanya E, Pfeiffer AFH, Johnsson E, Zhao J, Iqbal N, Bailey C. Metformin extended-release versus immediate-release: an international, randomized, double-blind, head-to-head trial in pharmacotherapy-naïve patients with type 2 diabetes. Diabetes Obes Metab. 2018;20:463-467.

11. March J, Silva S, Petrycki S, Curry J, Wells K, Fairbank J, et al. Fluoxetine, cognitive-behavioral therapy, and their combination for adolescents with depression: Treatment for Adolescents with Depression Study (TADS) randomized controlled trial. JAMA. 2004;292:807-820.

12. Treatment for Adolescents with Depression Study Team. Treatment for Adolescents with Depression Study (TADS): rationale, design, and methods. J Am Acad Child Adolesc Psychiatry. 2003:42:531-542.

13. The SPRINT Research Group. A randomized trial of intensive versus standard blood pressure control. N Engl J Med. 2015;373:2103-2116.

14. Miguel SR, Lima AF, Cruz LN, Cohen M, Zimmermann JJ, Ziegelmann PK, et al. Bipolar disorder mixed episodes: a pragmatic trial of a public health treatment effectiveness. Value in Health Regional Issues. 2018;17:158-163.

15. Talavera JO, Roy-García I, Palacios-Cruz L, Rivas-Ruiz R, Hoyo I, Pérez-Rodríguez M. De vuelta a la clínica. Métodos I. Diseños de investigación. Mayor calidad de información, mayor certeza a la respuesta. Gac Med Mex. 2019;155:399-405.

16. Feinstein AR. Clinical biostatistics. V. The architecture of clinical research (concluded). Clin Pharmacol Ther. 1970;11:755-771. 